\title{
Shape dependence of two-cylinder Rényi entropies for free bosons on a lattice
}

\author{
Leilee Chojnacki, ${ }^{1}$ Caleb Q. Cook, ${ }^{1}$ Denis Dalidovich, ${ }^{1}$ Lauren E. Hayward \\ Sierens, ${ }^{1,2, *}$ Étienne Lantagne-Hurtubise, ${ }^{1}$ Roger G. Melko, ${ }^{1,2}$ and Tiffany J. Vlaar ${ }^{1}$ \\ ${ }^{1}$ Perimeter Institute for Theoretical Physics, Waterloo, Ontario, N2L 2Y5, Canada \\ ${ }^{2}$ Department of Physics and Astronomy, University of Waterloo, Ontario, N2L 3G1, Canada
}

(Dated: October 20, 2018)

\begin{abstract}
Universal scaling terms occurring in Rényi entanglement entropies have the potential to bring new understanding to quantum critical points in free and interacting systems. Quantitative comparisons between analytical continuum theories and numerical calculations on lattice models play a crucial role in advancing such studies. In this paper, we exactly calculate the universal two-cylinder shape dependence of entanglement entropies for free bosons on finite-size square lattices, and compare to approximate functions derived in the continuum using several different ansatzes. Although none of these ansatzes are exact in the thermodynamic limit, we find that numerical fits are in good agreement with continuum functions derived using the AdS/CFT correspondence, an extensive mutual information model, and a quantum Lifshitz model. We use fits of our lattice data to these functions to calculate universal scalars defined in the thin-cylinder limit, and compare to values previously obtained for the free boson field theory in the continuum.
\end{abstract}

\section{INTRODUCTION}

Complementing traditional quantities used to understand critical phenomena such as scaling exponents, ${ }^{1}$ entanglement entropies have begun to provide physicists with a wealth of new quantities that exhibit universality across a wide variety of physical theories. Defined through a geometrical bipartition of a system into two parts $A$ and its complement $\bar{A}$, the entanglement entropy (and its generalized Rényi entropies) obey novel scaling behaviour as the size of $A$ is varied. The most famous example of a universal quantity extracted from such scaling is the central charge of a $(1+1)$-dimensional conformal field theory (CFT), which can be obtained by varying the length of $A \cdot^{2-5}$ In $d+1$ dimensions (for $d>1$ ) spatial geometries become highly non-trivial, and the entropy scaling is dominated by the ubiquitous "area" law, growing proportional to the boundary length between $A$ and $\bar{A} \cdot{ }^{6,7}$ The most useful universal numbers occur in scaling terms that are subleading to the area law. Like the central charge in $d=1$, these numbers can potentially give deep insight into the low-energy theories governing critical behaviour, providing for example an effective measure of degrees of freedom in the $\mathrm{CFT}^{8-12}$ or bounds on renormalization group flows, ${ }^{13-17}$ depending on the geometry of $A$. Furthermore, they can be calculated in a host of models and theories, such as non-interacting lattice models, interacting Hamiltonians tuned to a critical point, continuum CFTs, and strongly-interacting gravity duals through the $\mathrm{AdS} / \mathrm{CFT},{ }^{18}$ providing non-trivial insight into the correspondences between these different universal theories.

Just as critical exponents, particularly for interacting systems, often rely on lattice calculations such as series expansions or Monte Carlo for determination of their numerical values, so can lattice calculations provide access to universal numbers derived from entanglement entropy scaling. ${ }^{19}$ This approach has been important for interacting Hamiltonians. For example, quantum Monte Carlo (QMC) is able to access the integer $\alpha$ Rényi entropies on finite-size tori. ${ }^{20-24}$ Numerical linked cluster expansions (NLCE), combined with exact diagonalization ${ }^{25}$ or the density-matrix renormalization group

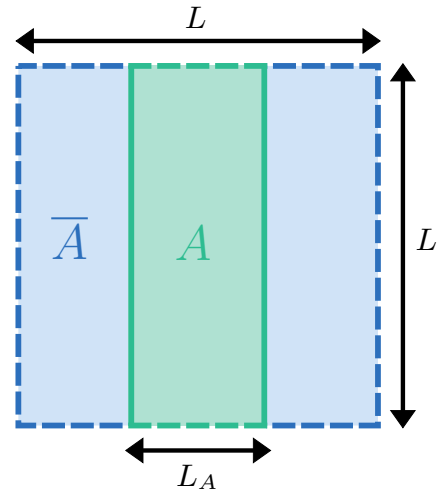

FIG. 1. An $L \times L$ square lattice divided in region $A$ and its complement, forming two cylinders. Each outer (dashed) boundary may be periodic or anti-periodic.

(DMRG), ${ }^{8,26}$ can provide finite-cluster-sized estimates for all $\alpha$ entropies in the thermodynamic limit. Lattice calculations have also proven important for accessing certain entangling geometries in free theories. ${ }^{27-31}$

In this paper, we focus on one specific geometry in $2+1$ dimensions: a torus cut into two cylinders. This is perhaps the most straightforward entanglement bipartition possible on a finite-size lattice, in that it avoids sharp corners (which induce a sub-leading logarithmic term), ${ }^{9,27,32,33}$ as well as curvatures in the boundary (which induce other sub-leading terms). ${ }^{34-37}$ In $d=2$, with a two-cylinder geometry defined as in Fig. 1, the Rényi entropies are expected to scale with the region $A$ as

$$
S_{\alpha}=a_{\alpha} \frac{L}{\delta}+\gamma_{\alpha}(u)+\cdots
$$

where $\delta$ is the lattice cutoff, $u=L_{A} / L, \alpha$ is the Rényi index (to be defined in Sec. III), and the ellipsis indicates nonuniversal constants and subleading terms depending on $\delta / L$ to some power. The function $\gamma(u)$ is not analytically known in closed form even for the simplest non-interacting theories, but it is expected to reflect universality. ${ }^{30}$ For a general lattice with linear sizes $L_{x}$ and $L_{y}$ respectively along the $x$ - 
and $y$-directions, $\gamma(u)$ also depends upon the aspect ratio ${ }^{30,38}$ $L_{x} / L_{y}$, but here we focus our attention on the case where $L_{x}=L_{y}=L$. In the thin-cylinder limit where $u \rightarrow 0$, this leading correction $\gamma(u)$ is expected to behave such that ${ }^{28,30}$

$$
\gamma_{\alpha}(u \rightarrow 0)=-\frac{\kappa_{\alpha}}{u},
$$

where $\kappa_{\alpha}$ is a universal constant.

Here, we numerically calculate $\gamma_{\alpha}(u)$ for all values of $u$ for non-interacting bosons on square lattices, and compare our results to several candidate functions derived from different ansatzes: the $(1+1)$-dimensional CFT scaling function, ${ }^{2-5}$ a quantum Lifshitz model (QLM), ${ }^{39}$ anti de-Sitter (AdS) gravity in $3+1$ dimensions ${ }^{38}$ and an extensive Mutual Information (EMI) model..$^{32,40-42}$ Using lattices of different size, we obtain data for the entanglement entropies $S_{\alpha}$ for free bosons, and explore the finite-size scaling behaviour of the residuals between the data and these candidate functions. We focus in particular on the von Neumann entropy $S_{1}$ and the second Rényi entropy $S_{2}$, though we find similar trends for other Rényi indices $\alpha>0$. Finally, we calculate the universal numbers $\kappa_{1}$ and $\kappa_{2}$ from fits of our numerical data to the QLM, AdS and EMI functions, and compare to values obtained from continuum ${ }^{28,32}$ and lattice calculations in the thincylinder limit.

\section{TWO-CYLINDER SCALING FUNCTIONS}

Attempts to understand the quantum critical Rényi entropy scaling of Fig. 1 through finite-size lattice numerics began with QMC simulations on interacting systems in $2+1$ dimensions. For a critical resonating-valence bond (RVB) wavefunction on a square lattice, Ju et al. ${ }^{43}$ postulated heuristically that the well-known $(1+1)$-dimensional CFT scaling function,

$$
\gamma_{1+1}(u)=c \ln \sin (\pi u),
$$

could apply for fixed $L$ (where $c$ is a constant that is proportional to the central change in $1+1$ dimensions). Subsequent examination on several interacting and non-interacting systems in $2+1$ dimensions show that, although this function works approximately, quantitative differences remain between it and finite-size lattice data extrapolated to the thermodynamic limit. ${ }^{22,38,43}$ For instance, $\gamma_{1+1}(u)$ does not obey Eq. (2) in the thin-cylinder limit.

An improvement on this form, motivated by the study of dimer RVB wavefunctions in the continuum limit, was derived by Stéphan et al. for the quantum Lifshitz model ${ }^{39}$ (QLM) with dynamical scaling exponent $z=2$. It is given by

$$
\gamma_{\mathrm{QLM}}(u)=\frac{24 \kappa}{\pi} \ln \left(\frac{\eta(2 i u) \eta(2 i(1-u))}{\theta_{3}(i \lambda u) \theta_{3}(i \lambda(1-u))}\right),
$$

sometimes called $J(u)$ in the literature. Here, $\theta_{3}$ is the Jacobi theta-function, $\eta$ is the Dedekind eta-function, and $\lambda$ is a model-dependent parameter. In the following, we fix $\lambda=2$ as for the dimer model in Ref. 39, although in principle this parameter could have a different value. Comparison of this function to finite-size scaling data on conformally-invariant $(z=1)$ critical points shows a surprisingly accurate fit, leading to the early speculation that it could be a universal scaling function relevant for all fixed points in $2+1$ dimensions, not just those specific to the QLM. ${ }^{22,38}$

$\mathrm{X}$. Chen et al. invoked the AdS/CFT correspondence to propose another candidate function, derived in $3+1$ dimensions using the AdS soliton metric. ${ }^{38}$ This holographic function is in a parameterized form; up to a constant, it is (for an $L \times L$ lattice),

$\gamma_{\mathrm{AdS}}(\chi)=\frac{\kappa \Gamma^{4}\left(\frac{1}{4}\right)}{3 \pi^{2}} \chi^{-1 / 3}\left(\int_{0}^{1} \frac{d \zeta}{\zeta^{2}}\left(\frac{1}{\sqrt{P(\chi, \zeta)}}-1\right)-1\right)$

where $P(\chi, \zeta)=1-\chi \zeta^{3}-(1-\chi) \zeta^{4}$ and $\Gamma$ is the gamma function. The parameter $\chi$ is related to the aspect ratio $u=$ $L_{A} / L$ through the equation

$$
u(\chi)=\frac{3 \chi^{1 / 3}(1-\chi)^{1 / 2}}{2 \pi} \int_{0}^{1} \frac{d \zeta \zeta^{2}}{\left(1-\chi \zeta^{3}\right)} \frac{1}{\sqrt{P(\chi, \zeta)}} .
$$

Numerically, this function appears to describe very well the subleading entropy scaling term of two scale-invariant fermionic models: free massless Dirac fermions and a model of fermions with quadratic band touching. ${ }^{38}$

Most recently, Witczak-Krempa et al. used an extensive mutual information (EMI) model, ${ }^{32,40-42}$ to derive another functional form, ${ }^{30}$

$$
\gamma_{\mathrm{EMI}}(u)=\frac{2 \kappa}{\pi}\left[\frac{\operatorname{arccot}(2 u)}{u}+\frac{\operatorname{arccot}(2(1-u))}{1-u}\right] .
$$

The EMI model has been shown to be useful in the analysis of entropy scaling in CFTs ${ }^{9,30,32}$.

In the next section, we introduce our calculation for the free boson field theory on a finite-size lattice, and use numerical solutions for the Rényi entropies to evaluate each of the candidate scaling functions outlined above.

\section{FREE BOSONS ON THE SQUARE LATTICE}

Beginning with the action for a free real scalar (KleinGordon field) $\phi$ of mass $m$ in $d+1$ dimensions, one can regularize the theory on a finite two-dimensional square lattice, such that the field and its conjugate momentum $\pi_{i}$ exist at each lattice site $i$ and evolve according to the Hamiltonian

$$
\begin{gathered}
H=\frac{1}{2} \sum_{x, y=1,1}^{L_{x}, L_{y}}\left[\pi_{x, y}^{2}+\left(\phi_{x+1, y}-\phi_{x, y}\right)^{2}+\left(\phi_{x, y+1}-\phi_{x, y}\right)^{2}\right. \\
\left.+m^{2} \phi_{x, y}^{2}\right] .
\end{gathered}
$$

Here, $L_{x}$ and $L_{y}$ are the linear dimensions of the lattice, and the total number of sites is $N=L_{x} \times L_{y}$. Transforming into Fourier space, the Hamiltonian can be written in the form of 
$N$ uncoupled simple harmonic oscillators,

$$
H=\frac{1}{2} \sum_{\mathbf{k}}\left[\pi_{\mathbf{k}} \pi_{-\mathbf{k}}+\omega_{\mathbf{k}}^{2} \phi_{\mathbf{k}} \phi_{-\mathbf{k}}\right]
$$

where

$$
\omega_{\mathbf{k}}=\sqrt{4 \sin ^{2}\left(k_{x} / 2\right)+4 \sin ^{2}\left(k_{y} / 2\right)+m^{2}} .
$$

The ground-state two-point correlation functions are given in Fourier space by

$$
\begin{aligned}
\left\langle\phi_{\mathbf{k}} \phi_{-\mathbf{k}^{\prime}}\right\rangle & =\frac{1}{2 \omega_{\mathbf{k}}} \delta_{\mathbf{k k}^{\prime}}, \\
\left\langle\pi_{\mathbf{k}} \pi_{-\mathbf{k}^{\prime}}\right\rangle & =\frac{\omega_{\mathbf{k}}}{2} \delta_{\mathbf{k k}^{\prime}} .
\end{aligned}
$$

These correlation functions can be transformed back to real space, and by restricting the numbers of lattice points, the momenta are quantized. On a translationally invariant lattice, we thus obtain

$$
\begin{aligned}
\left\langle\phi_{\mathbf{x}} \phi_{\mathbf{x}^{\prime}}\right\rangle & =\frac{1}{2 L_{x} L_{y}} \sum_{\mathbf{k}} \frac{\cos \left[k_{x}\left(x-x^{\prime}\right)\right] \cos \left[k_{y}\left(y-y^{\prime}\right)\right]}{\omega_{\mathbf{k}}} \\
\left\langle\pi_{\mathbf{x}} \pi_{\mathbf{x}^{\prime}}\right\rangle & =\frac{1}{2 L_{x} L_{y}} \sum_{\mathbf{k}} \omega_{\mathbf{k}} \cos \left[k_{x}\left(x-x^{\prime}\right)\right] \cos \left[k_{y}\left(y-y^{\prime}\right)\right] .
\end{aligned}
$$

If one considers periodic boundary conditions (PBC) for the fields, the momentum sums are restricted to $k_{i}=2 n_{i} \pi / L_{i}$, where $n_{i}=0,1, \ldots, L_{i}-1$. Note that the case where the boson is massless, $m=0$, is desired in order to obtain a scaleinvariant critical theory, but the presence of the zero-mode $k_{x}=k_{y}=0$ for PBC in this case causes the $\phi$ correlation function to diverge. The entanglement entropy thus cannot be calculated directly for a fully periodic system in the massless (critical) case, and for such systems we include a small but finite mass of $m=10^{-6}$. Alternatively, in many of the calculations below, we will employ an anti-periodic boundary condition (APBC), e.g. $\phi_{x}=-\phi_{x+L_{x}}$, in at least one lattice direction, which gives $k_{i}=\left(2 n_{i}+1\right) \pi / L_{i}$ and avoids this divergence (even when $m=0$ ).

We employ these correlation functions to calculate the von Neumann entanglement entropy, defined in terms of the reduced density matrix $\rho_{A}=\operatorname{Tr}_{B}\left(\rho_{A B}\right)$ as

$$
S_{1}(A)=-\operatorname{Tr}\left(\rho_{A} \log \rho_{A}\right) .
$$

We also study the generalized Rényi entanglement entropies, which are given by

$$
S_{\alpha}(A)=\frac{1}{1-\alpha} \log \left(\operatorname{Tr} \rho_{A}^{\alpha}\right),
$$

where $\alpha$ is called the Rényi index. The case of $\alpha=2$ is particularly important for QMC studies of strongly-interacting lattice Hamiltonians. ${ }^{20}$ Note that taking the limit $\alpha \rightarrow 1$ in Eq. (15) recovers the (von Neumann) entanglement entropy of Eq. (14).
As discussed by Peschel in Ref. 44 and by Casini and Huerta in Ref. 28, the reduced density matrix for a noninteracting system such as this one can be written as $\rho_{A}=$ $\mathcal{K} e^{-H_{A}}$, where $\mathcal{K}$ is a normalization constant and $H_{A}$ is called the modular Hamiltonian, which is quadratic and acts only on sites in region $A$. The von Neumann and Rényi entropies can be calculated from the correlation functions within region $A$, thus avoiding the trace over $\bar{A}$ in the calculation of the reduced density matrix. Defining the elements of our correlation matrices $X_{A}$ and $P_{A}$ such that $\left(X_{A}\right)_{i j}=\left\langle\phi_{\mathbf{x}_{\mathbf{i}}} \phi_{\mathbf{x}_{\mathbf{j}}}\right\rangle$ and $\left(P_{A}\right)_{i j}=\left\langle\pi_{\mathbf{x}_{\mathbf{i}}} \pi_{\mathbf{x}_{\mathbf{j}}}\right\rangle$ for sites $\mathbf{x}_{\mathbf{i}}$ and $\mathbf{x}_{\mathbf{j}}$ in region $A$, the entropies are then given in terms of the eigenvalues $\nu_{\ell}$ of the matrix $C_{A}=\sqrt{X_{A} P_{A}}$ by

$$
\begin{aligned}
S_{1}(A)=\sum_{\ell}[ & \left(\nu_{\ell}+\frac{1}{2}\right) \log \left(\nu_{\ell}+\frac{1}{2}\right) \\
& \left.-\left(\nu_{\ell}-\frac{1}{2}\right) \log \left(\nu_{\ell}-\frac{1}{2}\right)\right],
\end{aligned}
$$

and

$$
S_{\alpha}(A)=\frac{1}{\alpha-1} \sum_{\ell} \log \left[\left(\nu_{\ell}+\frac{1}{2}\right)^{\alpha}-\left(\nu_{\ell}-\frac{1}{2}\right)^{\alpha}\right] .
$$

The procedure described above allows us to calculate the two-cylinder entropy $S_{\alpha}(u)$ for the full range of $u$ values for lattice sizes up to $L=240$ using modest computational resources. In addition, for the two-cylinder geometry discussed in this paper, we employ an extension of the above arguments as given in Ref. 38 that takes advantage of the translational symmetry in one lattice direction to map the $(2+1)$ dimensional model to an effective model consisting of $L$ separate $(1+1)$-dimensional chains. This mapping allows for the calculation of Rényi entropies on significantly larger lattices.

\section{RESULTS}

We use the procedure of the last section to calculate the von Neumann $\left(S_{1}\right)$ and second Rényi $\left(S_{2}\right)$ entropies for lattice geometries of size $L \times L$, with $L_{A}$ varying from 1 to $L / 2$ (see Fig. 1). An example of a finite-size lattice calculation of $S_{1}$ as a function of $u$, for a fixed value of $L$, is illustrated in Fig. 2 .

For fixed $L$, the area law and subleading terms in Eq. (1) are constant such that, as a function of $u$, we have $S_{\alpha}(u)=$ $\gamma_{\alpha}(u)+d_{\alpha}$, where $d_{\alpha}$ is a constant ( $L$-dependent) parameter and the function $\gamma_{\alpha}(u)$ contains the additional parameter $\kappa_{\alpha}$ (or $c_{\alpha}$ for $\gamma_{1+1}$ ). In order to reduce the number of fitting parameters from two to one, we perform least-squares fits of $S_{\alpha}(1 / 2)-S_{\alpha}(u)$ to the form $\gamma_{\alpha}(1 / 2)-\gamma_{\alpha}(u)$ such that $\kappa_{\alpha}$ (or $c_{\alpha}$ ) becomes the sole fitting parameter. Fig. 2 illustrates such fits for the four candidate functions for $\gamma_{\alpha}(u)$ from Eqs. (3), (4), (5) and (7) for the case where $\alpha=1$. From this, it is immediately obvious that $\gamma_{1+1}$ provides a poor approximation to the data. The inset shows the amount that the three best fits deviate from the free boson as a function of $u$.

While this procedure provides the deviation of the free boson from the candidate functions for a given finite-size lattice, 


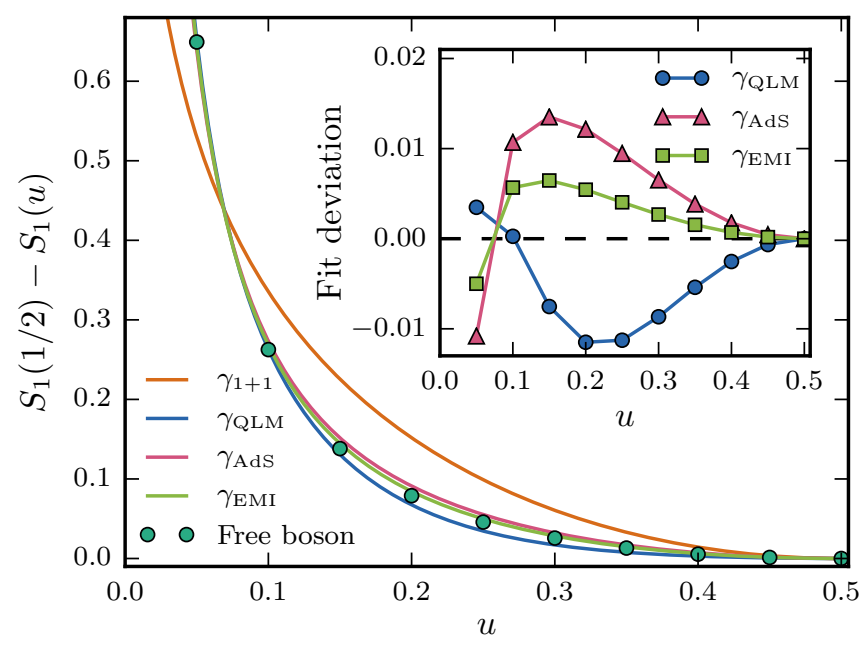

FIG. 2. Shape dependence of the von Neumann entanglement entropy for an $L=3000$ free boson system with PBC along the $x$ direction and $\mathrm{APBC}$ along the $y$-direction, and fits corresponding to the four candidate functions discussed in Sec. II (each with resolution $\Delta u=0.05$ ). The inset illustrates the amount that each fit deviates from each data point. The fit deviation for $\gamma_{1+1}$ is excluded since it is much larger in magnitude than the other three curves.

one may ask how this deviation behaves as one scales the lattice size towards the thermodynamic limit. Thus, we vary the system size and repeat the calculation for a range of values of $L$. In order to quantify the goodness of each one-parameter fit, we sum the squared residuals and normalize by the number of degrees of freedom, defining the fitting error by

$E_{\alpha}=\frac{1}{n_{u}-2} \sum_{i=1}^{n_{u}}\left(\left[\gamma_{\alpha}(1 / 2)-\gamma_{\alpha}\left(u_{i}\right)\right]-\left[S_{\alpha}(1 / 2)-S_{\alpha}\left(u_{i}\right)\right]\right)^{2}$.

Here, the data points $S_{\alpha}\left(u_{i}\right)$ are the calculated free boson entanglement entropies. The fitted function is $\gamma_{\alpha}\left(u_{i}\right)$, with fitting parameter $\kappa_{\alpha}$ (or $c_{\alpha}$ in $d=1$ ). Finally, $n_{u}$ is the number of values of $u$ used within the fitting procedure.

In doing these fits, we find that the errors are especially sensitive to the data points at small $u$. In particular, if the fitting procedure uses all $L / 2$ available data points, then the errors appear to diverge as the lattice size $L$ increases. However, this divergence can be attributed to the fact that the resolution of a lattice scales according to $\Delta u=1 / L$, and thus larger lattices are capable of probing smaller values of $u$. Since these small$u$ effects are not what we wish to measure, we perform our fits using a resolution $\Delta u$ and corresponding number of data points $n_{u}=1 /(2 \Delta u)$ that remain fixed as the lattice size $L$ increases. Such a constraint limits the lattice sizes on which we perform our fits to multiples of $1 / \Delta u$.

One can employ this type of fixed-resolution fitting approach to evaluate a $(1+1)$-dimensional free boson system, which is known to exactly obey the scaling function in Eq. (3) in the thermodynamic limit. As shown in Fig. 3, we indeed find for free bosons in $1+1$ dimensions that the fitting errors corresponding to Eq. (3) trend to zero (within machine precision) for PBC, validating our use of Eq. (18) to quantify the

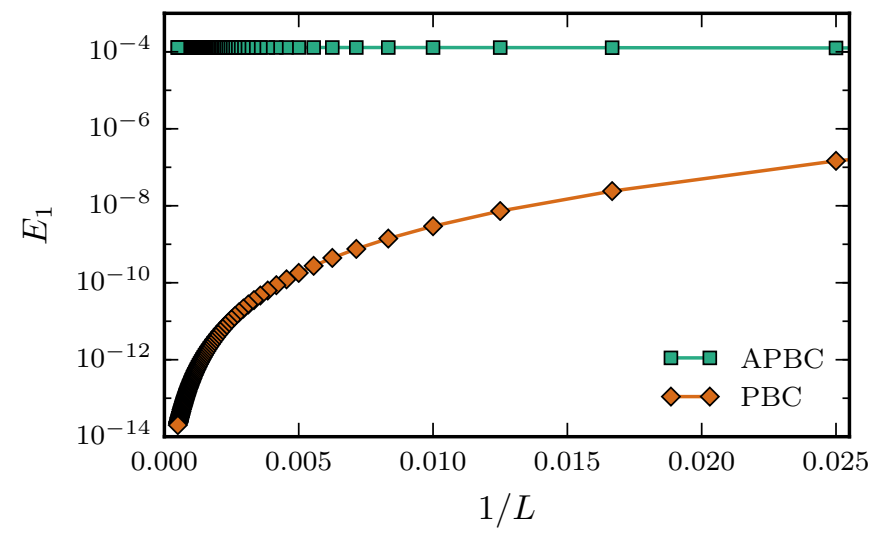

FIG. 3. The fitting errors corresponding to fits of the $(1+1)$ dimensional free boson von Neumann entropies to the expression in Eq. (3). We observe that this expression is exact in the thermodynamic limit (i.e. the errors trend to zero) for PBC but not for APBC. These errors are measured using resolution $\Delta u=0.05$ on lattices of size $L=40,60, \ldots, 2000$. For the periodic case, we set $m=10^{-6}$ as explained in the text.

residuals. Interestingly, for APBC, the fitting error does not disappear in the limit $L \rightarrow \infty$, indicating that Eq. (3) is not the correct description of the entanglement correction $\gamma_{\alpha}$ for this boundary condition.

In $2+1$ dimensions, the errors $E_{\alpha}$ have different finite-size scaling trends for the different candidate functional forms of $\gamma_{\alpha}$, as illustrated in Fig. 4. We find that the behaviour of the error trends also depend both upon the chosen resolution $\Delta u$ and upon the lattice boundary conditions. PBC along the $x$ direction and APBC along the $y$-direction are used to generate the plots presented in Figs. 2, 4 and 5. Table I summarizes the fitting errors measured using resolution $\Delta u=0.05$ for various boundary conditions. For the von Neumann entropy $S_{1}$, the functions $\gamma_{\mathrm{QLM}}$ and $\gamma_{\mathrm{EMI}}$ consistently yield the lowest fitting errors out of the four candidate functions, once at least one boundary is anti-periodic. It is interesting that for the case of periodic boundary conditions in both directions, the fitting errors for $\gamma_{\mathrm{QLM}}, \gamma_{\mathrm{EMI}}$ and $\gamma_{\mathrm{AdS}}$ all become much larger, while for $\gamma_{1+1}$ these errors change only slightly and no longer correspond to the worst fit. The explanation of this behaviour may lie in the effects of additional subleading scaling terms, which are induced by the presence of a finite mass $m,{ }^{45}$ on each of these fits. In the case of the second Rényi entropy, the errors corresponding to $\gamma_{\mathrm{QLM}}$ are consistently lowest, except (again) for the case of PBC in both directions.

In addition to exploring the most suitable functional form for $\gamma_{\alpha}(u)$ for $\alpha=1$ and 2, we also examine the ability of our fits to extract universal numbers such as the coefficient $\kappa_{\alpha}$. For $(2+1)$-dimensional massless real free bosons in the continuum, this coefficient $\kappa_{1}$ has been calculated numerically for the von Neumann entropy ${ }^{28}$ to be $\kappa_{1}=0.0397$ as well as for the second Rényi entropy ${ }^{32}$ to be $\kappa_{2}=0.0227998$. On a lattice, one can calculate $\kappa_{\alpha}$ by fitting to $\gamma(u \rightarrow 0)$ in Eq. (2) for small $u$. Here we use a slightly different procedure than the fixed-resolution approach used to fit the four candidate functions: for a given lattice of size $L \geq 80$, we extract $\kappa_{\alpha}$ in 

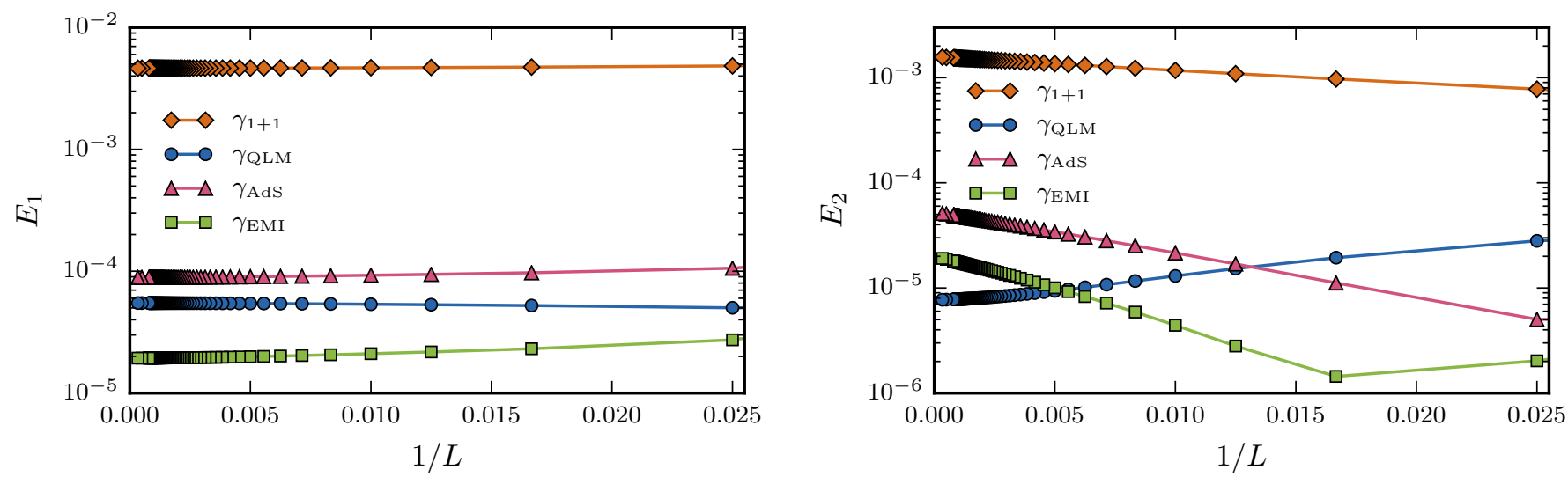

FIG. 4. The fitting errors corresponding to the four candidate functions for $\gamma$ as a function of $1 / L$ for the $2 \mathrm{D}$ free boson von Neumann (left) and second Rényi (right) entropies. Both plots have PBC along the $x$ direction and APBC along the $y$ direction. The errors are measured using resolution $\Delta u=0.05$ on lattices of linear size $L=40$ up to 3000 , as explained in the main text.

\begin{tabular}{|c|c|c|c|c|c|c|}
\hline Fitting error & $x$-direction & $y$-direction & $1+1$ & QLM & AdS & EMI \\
\hline \multirow{3}{*}{$E_{1}$} & PBC & PBC & $3.18 \times 10^{-3}$ & $4.13 \times 10^{-3}$ & $1.56 \times 10^{-3}$ & $2.25 \times 10^{-3}$ \\
& PBC & APBC & $4.63 \times 10^{-3}$ & $5.49 \times 10^{-5}$ & $8.90 \times 10^{-5}$ & $1.94 \times 10^{-5}$ \\
& APBC & PBC & $4.60 \times 10^{-3}$ & $4.24 \times 10^{-5}$ & $1.12 \times 10^{-4}$ & $3.27 \times 10^{-5}$ \\
& APBC & APBC & $5.05 \times 10^{-3}$ & $2.85 \times 10^{-5}$ & $3.10 \times 10^{-4}$ & $1.69 \times 10^{-4}$ \\
\hline \multirow{3}{*}{$E_{2}$} & PBC & PBC & $9.28 \times 10^{-4}$ & $2.61 \times 10^{-3}$ & $1.19 \times 10^{-3}$ & $1.59 \times 10^{-3}$ \\
& PBC & APBC & $1.56 \times 10^{-3}$ & $7.73 \times 10^{-6}$ & $5.12 \times 10^{-5}$ & $1.91 \times 10^{-5}$ \\
& APBC & PBC & $1.51 \times 10^{-3}$ & $9.94 \times 10^{-6}$ & $4.68 \times 10^{-5}$ & $1.70 \times 10^{-5}$ \\
& APBC & APBC & $1.67 \times 10^{-3}$ & $2.06 \times 10^{-5}$ & $1.35 \times 10^{-4}$ & $8.28 \times 10^{-5}$ \\
\hline
\end{tabular}

TABLE I. The fitting errors corresponding to the four candidate functions and different boundary conditions for the von Neumann and second Rényi entropies. The errors in this table are measured using resolution $\Delta u=0.05$ on a square lattice of size $L=3000$. Calculations for the fully periodic system include a small mass $m=10^{-6}$.

the $u \rightarrow 0$ limit from our free boson calculations by fitting $S_{\alpha}(40 / L)-S_{\alpha}(u)$ to Eq. (2) for $u=31 / L, 32 / L, \ldots, 40 / L$ (we ignore the smallest 30 values of $u$ due to numerical issues that arise when the cylinder becomes very thin). Results are illustrated in Fig. 5.

In addition, the QLM, AdS and EMI functions all obey Eq. (2) in the small- $u$ limit, allowing for predictions of the universal number $\kappa$ from each. In Fig. 5 we illustrate the $\kappa_{1}$ and $\kappa_{2}$ coefficients as predicted from fits (for the entire range of $u$ values and with fitting resolution $\Delta u=0.05$ ) to $\gamma_{\mathrm{QLM}}$, $\gamma_{\mathrm{AdS}}$ and $\gamma_{\mathrm{EMI}}$. Although we know from the fitting errors that none of these three candidate functions are exact in the thermodynamic limit, they are all still capable of extracting estimates for $\kappa_{\alpha}$ that agree relatively well with the previouslycalculated continuum values ${ }^{28,32}$ and the lattice values from fits to Eq. (2). In particular, $\gamma_{\mathrm{QLM}}$ and $\gamma_{\mathrm{EMI}}$ both yield estimates for $\kappa_{1}\left(\kappa_{2}\right)$ that are within less than $5 \%(9 \%)$ of the value calculated in Ref. 28 (Ref. 32).

\section{DISCUSSION}

In this paper, we have studied the shape-dependence of the bipartite Rényi entanglement entropies for a system of free bosons in $2+1$ dimensions. Of particular interest is the universal term $\gamma_{\alpha}(u)$ that occurs sub-leading to the area law, which depends on the ratio $u$ of the length of an entangled cylindrical region $A$ to the length of the entire system. For this and other CFTs, an analytical expression for this universal function is unknown. However, several candidate functions have been used in the literature to approximate $\gamma_{\alpha}(u)$. Performing exact but finite-size lattice calculations for free bosons on a $(2+1)$-dimensional square lattice, we evaluate the quality of several of these candidate functions in the limit of large lattice size by examining a fitting error between the data and each respective function.

We concentrate mainly on the von Neumann $\left(S_{1}\right)$ and second Rényi $\left(S_{2}\right)$ entropies. For each of these quantities, the candidate function derived heuristically from the known $(1+$ 1)-dimensional CFT scaling form performs poorly and does not produce the correct thin-cylinder behaviour of Eq. (2), indicating that it should not be used to approximate $(2+1)$ - 

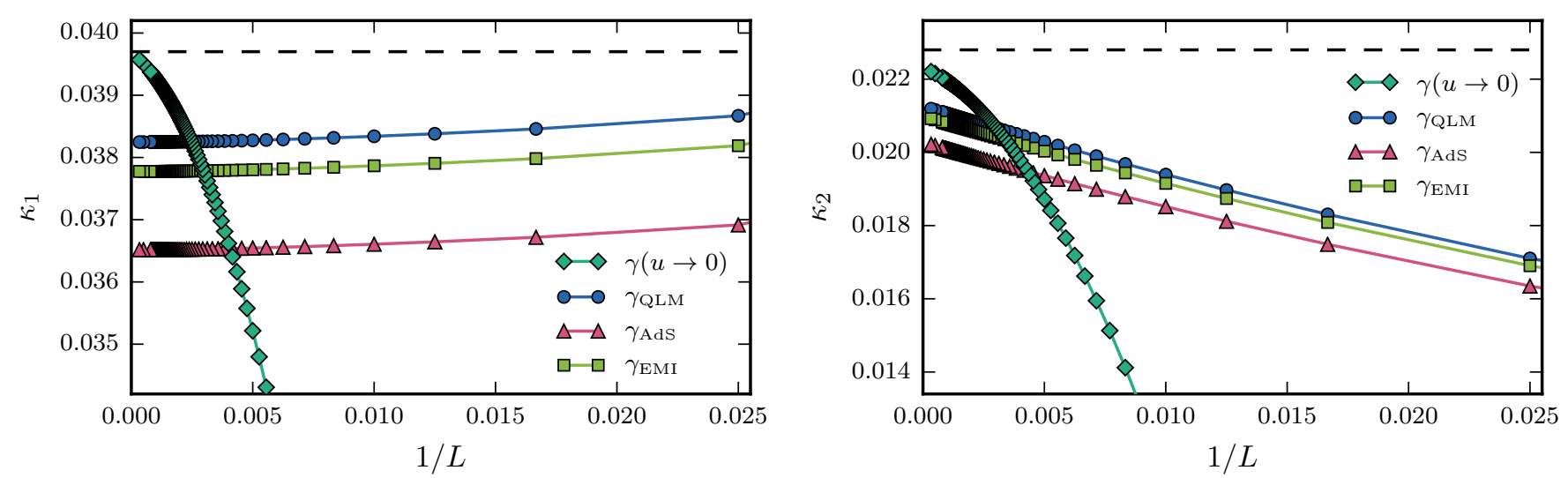

FIG. 5. The universal number $\kappa_{\alpha}$ for the von Neumann (left) and second Rényi (right) entropies, as extracted from fits to $\gamma(u \rightarrow 0), \gamma_{\mathrm{QLM}}$, $\gamma_{\mathrm{AdS}}$ and $\gamma_{\mathrm{EMI}}$ (as described in the main text). The dashed lines indicate the values calculated in the continuum in the $u \rightarrow 0$ limit ${ }^{28,32}$.

dimensional CFT data in the two-cylinder geometry. Three other candidate functions with the correct behaviour in the $u \rightarrow 0$ limit were examined, derived from: a quantum Lifshitz model (QLM), anti de-Sitter (AdS) gravity in $3+1$ dimensions, and an extensive Mutual Information (EMI) model. All three of these give quantitatively better fits when compared to the $(1+1)$-dimensional CFT form. Worst performing is the AdS function. The QLM and EMI provide the best fits. The success of the QLM is perhaps surprising: it has an additional parameter $\lambda$ that is unknown and has been fixed to an arbitrary value in our fits, and it is derived in a non-conformally invariant theory with dynamical exponent $z=2$. Despite there being no theoretical reason to believe it should apply to our boson CFT, which has $z=1$, it describes our data relatively well. Finally, we note that the quantitative error in the fits is significantly affected by different combinations of periodic and anti-periodic boundary conditions, indicating that the exact CFT function $\gamma_{\alpha}(u)$, whatever it is, will also depend on the phase angle by which the field $\phi$ is twisted at the boundary.

This work illustrates the care that must be taken when making comparisons of analytical functions, derived in the continuum limit, to exact but finite-size entropy data obtained for lattice models. Not only are the residuals of fits to such functions affected by the finite size of the lattice itself, but also on the range and position of data chosen for the comparison. Nevertheless, with sufficient care, the synergy between continuum theories and lattice numerics can bear fruit, such as we have demonstrated with the extraction of the universal coefficients $\kappa_{\alpha}$. The ability to calculate and compare such universal scalar quantities in the lattice and the continuum is of crucial importance in the continuing effort to use entanglement entropies as tools to characterize both free and strongly-interacting quantum critical points.

\section{ACKNOWLEDGMENTS}

We acknowledge crucial discussions with X. Chen, P. Fendley, E. Fradkin, A. Ludwig, M. Metlitski, J.-M. Stéphen, G. Vidal and W. Witczak-Krempa. We appreciate the hospitality of the organizers of the Perimeter Institute Winter School, where this project was initially conceived, and the Aspen Center for Physics, where it was completed. The simulations were performed on the computing facilities of SHARCNET. L.H.S. gratefully acknowledges funding from the Ontario Graduate Scholarship, and É. L.-H. is partially funded by FRQNT. Support was provided by NSERC, the Canada Research Chair program, the Ontario Ministry of Research and Innovation, and the Perimeter Institute for Theoretical Physics. Research at Perimeter Institute is supported by the Government of Canada through the Department of Innovation, Science and Economic Development Canada and by the Province of Ontario through the Ministry of Research, Innovation and Science. *1hayward@perimeterinstitute.ca

${ }^{1}$ H. E. Stanley, Rev. Mod. Phys. 71, S358 (1999).

${ }^{2}$ C. Holzhey, F. Larsen, and F. Wilczek, Nucl. Phys. B 424, 443 (1994).

3 G. Vidal, J. I. Latorre, E. Rico, and A. Kitaev, Phys. Rev. Lett. 90, 227902 (2003).

${ }^{4}$ V. E. Korepin, Phys. Rev. Lett. 92, 096402 (2004).

5 P. Calabrese and J. Cardy, Journal of Statistical Mechanics: Theory and Experiment 2004, P06002 (2004).
${ }^{6}$ L. Bombelli, R. K. Koul, J. Lee, and R. D. Sorkin, Phys. Rev. D 34, 373 (1986).

${ }^{7}$ M. Srednicki, Phys. Rev. Lett. 71, 666 (1993).

8 A. B. Kallin, E. M. Stoudenmire, P. Fendley, R. R. P. Singh, and R. G. Melko, Journal of Statistical Mechanics: Theory and Experiment 2014, P06009 (2014).

9 P. Bueno, R. C. Myers, and W. Witczak-Krempa, Phys. Rev. Lett. 115, 021602 (2015).

10 R.-X. Miao (2015), arXiv:1507.06283. 
11 P. Bueno and R. C. Myers, Journal of High Energy Physics 2015, 1 (2015).

12 T. Faulkner, R. G. Leigh, and O. Parrikar, Journal of High Energy Physics 2016, 1 (2016).

13 A. Zamolodchikov, JETP Lett. 43, 730 (1986).

14 J. L. Cardy, Physics Letters B 215, 749 (1988), ISSN 0370-2693.

15 H. Casini, M. Huerta, and R. Myers, Journal of High Energy Physics 2011, 36 (2011).

${ }^{16}$ I. R. Klebanov, T. Nishioka, S. S. Pufu, and B. R. Safdi, Journal of High Energy Physics 2012 (2012).

17 T. Grover, Phys. Rev. Lett. 112, 151601 (2014).

18 S. Ryu and T. Takayanagi, Phys. Rev. Lett. 96, 181602 (2006).

19 R. R. P. Singh, R. G. Melko, and J. Oitmaa, Phys. Rev. B 86, 075106 (2012).

20 M. B. Hastings, I. González, A. B. Kallin, and R. G. Melko, Phys. Rev. Lett. 104, 157201 (2010).

21 S. Humeniuk and T. Roscilde, Phys. Rev. B 86, 235116 (2012).

22 S. Inglis and R. G. Melko, New Journal of Physics 15, 073048 (2013).

23 J. Helmes and S. Wessel, Phys. Rev. B 89, 245120 (2014).

24 J. Helmes and S. Wessel, Phys. Rev. B 92, 125120 (2015).

25 A. B. Kallin, K. Hyatt, R. R. P. Singh, and R. G. Melko, Phys. Rev. Lett. 110, 135702 (2013).

${ }^{26}$ E. M. Stoudenmire, P. Gustainis, R. Johal, S. Wessel, and R. G. Melko, Phys. Rev. B 90, 235106 (2014).

27 H. Casini and M. Huerta, Nuclear Physics B 764, 183 (2007).

${ }^{28}$ H. Casini and M. Huerta, Journal of Physics A: Mathematical and Theoretical 42, 504007 (2009).
29 S. Sahoo, E. M. Stoudenmire, J.-M. Stéphan, T. Devakul, R. R. P. Singh, and R. G. Melko, Phys. Rev. B 93, 085120 (2016).

${ }^{30}$ W. Witczak-Krempa, L. E. Hayward Sierens, and R. G. Melko (2016), arXiv:1603.02684.

31 J. Helmes, L. E. Hayward Sierens, A. Chandran, W. WitczakKrempa, and R. G. Melko (2016), arXiv:1606.03096.

${ }^{32}$ P. Bueno, R. C. Myers, and W. Witczak-Krempa, Journal of High Energy Physics 2015 (2015).

${ }^{33}$ P. Bueno and W. Witczak-Krempa, Phys. Rev. B 93, 045131 (2016).

${ }^{34}$ H. Casini and M. Huerta, Phys. Rev. D 85, 125016 (2012).

35 H. Liu and M. Mezei, Journal of High Energy Physics 2013, 1 (2013), ISSN 1029-8479.

${ }^{36}$ H. Casini, M. Huerta, R. C. Myers, and A. Yale, Journal of High Energy Physics 2015, 1 (2015), ISSN 1029-8479.

37 T. Zhou, X. Chen, T. Faulkner, and E. Fradkin (2016), arXiv:1607.01771.

38 X. Chen, G. Y. Cho, T. Faulkner, and E. Fradkin, Journal of Statistical Mechanics: Theory and Experiment 2015, P02010 (2015).

39 J.-M. Stéphan, H. Ju, P. Fendley, and R. G. Melko, New. J. Phys. 15, 015004 (2013).

${ }^{40}$ H. Casini, C. D. Fosco, and M. Huerta, Journal of Statistical Mechanics: Theory and Experiment 2005, P07007 (2005).

${ }^{41}$ H. Casini and M. Huerta, Journal of High Energy Physics 2009, 048 (2009).

${ }^{42}$ B. Swingle (2010), arXiv:1010.4038.

${ }^{43}$ H. Ju, P. Kallin, Ann B.Fendley, M. B. Hastings, and R. G. Melko, Phys. Rev. B 85, 165121 (2012).

44 I. Peschel, J. Phys. A: Math. Gen. 36, L205 (2003).

45 M. A. Metlitski and T. Grover (2011), arXiv:1112.5166. 